Retrospective Evaluation

\title{
e Initial Clinical Outcomes of Percutaneous Full- Endoscopic Lumbar Discectomy Using an Interlaminar Approach at the L4-L5
}

\author{
Jun-ichiro Nakamura, MD, and Kiyoshi Yoshihara, MD
}

From: Kawasaki Saiwai Hospital Kawasaki, Kanagawa Prefecture, Japan

Address Correspondence: Jun-ichiro Nakamura, MD Kawasaki-Saiwai Hospital Orthopedic surgery 31-27 OhmiyaSaiwai-ku Kawasaki, Kanagawa prefecture 212-0014, Japan Email:

nakajun16@yahoo.co.jp

Disclaimer: There was no external funding in the preparation of this manuscript. Conflict of interest: Each author certifies that he or she, or a member of his or her immediate

family, has no commercial association (i.e., consultancies, stock ownership, equity interest, patent/licensing arrangements, etc.) that might pose a conflict of interest in connection with the submitted manuscript.

Manuscript received: 03-21-2016

Revised manuscript received: o8-11-2016

Accepted for publication: 11-14-2016

Free full manuscript: www.painphysicianjournal.com
Background: Percutaneous full-endoscopic discectomy (PED) is being increasingly used because of its potential to minimalize soft-tissue damage and decrease hospital stay. PED using the interlaminar approach (PED-IL) at L4-L5 is performed by only a few surgeons. To the best of our knowledge, the safety and efficacy of PED-IL at L4-L5, without experience in PED via a transforaminal approach (PED-TF) has not been previously reported.

Objective: This study aimed to evaluate initial clinical outcomes and complications of PED-IL at L4-L5 without experience in PED-TF.

Study Design: Retrospective evaluation.

Setting: An urban minimally invasive spine hospital.

Methods: Of a total of 50 patients (36 men and 14 women, ages ranging from 21-59 years, with the average age being 40.3 years old), 16 cases were performed at L4-L5 and 34 cases were performed at L5-S1. PED-IL was successfully completed in all cases, and no case required conversion to open surgery. The operative time, hospital stay, modified MacNab criteria, and visual analog scale (VAS) scores were examined at L5-S1 (range: 41-112). There was no significant difference in operative time between the L4-L5 and L5-S1 groups; the operative time was gradually decreased. The mean hospital stay was 2.9 days (range: 2-8 days). According to modified MacNab criteria, 20 cases (6 at L4-L5 and 14 at L5-S1) were excellent, 27 (10 at L4-L5 and 17 at L5-S1) were good, one at L5-S1 was fair, and 2 at L5-S1 were poor. Two perineurium tears occurred at L5-S1. There was no infection or recurrence of herniated nucleus pulposus (HNP). The clinical outcomes of PED-IL at L4-L5 were equal to those at L5- S1.

Results: The mean operative time was $71.3 \pm 19.3$ minutes for all cases (range: $41-112$ mins.), 76.1 \pm 16.8 minutes at L4-L5 (range: 52-102 mins.), and 70.5 \pm 20.1 minutes at L5-S1 (range: 41-112 mins.).

Limitations: A small sample size and a short follow-up period.

Conclusions: The clinical outcomes of PED-IL at L4-L5 were equal to those at L5-S1. Therefore, PED-IL is suitable to be a standard method for any type of intracanalicular disc herniation.

Keywords: Percutaneous full-endoscopic lumbar discectomy, interlaminar approach, clinical outcome, herniated nucleus pulpous, MacNab criteria, intracanalicular disc

herniation, learning curve

Pain Physician 2017; 20:E507-E512 ndoscopic lumbar discectomy was developed over 2 decades ago as a minimally invasive approach for spinal surgery. Recently, percutaneous endoscopic discectomy (PED) is being increasingly used worldwide (1). PED via a transforaminal approach (PED-TF) is a popular procedure among endoscopic spine surgeons because it is easy to access the disc, does not require ligamentum flavum or lamina removal, 
and can be performed under local anesthesia (2). Despite advancements in endoscopic techniques and equipment, several surgeons continue to have concerns regarding incomplete herniated disc removal using PED-TF. Furthermore, PED-TF is sometimes difficult to perform because of anatomical variations, such as a high iliac wing for L5-S1 or migration of a herniated nucleus pulposus (HNP) (3). In such situations, PED via an interlaminar approach (PED-IL) is useful, particularly at L5-S1 $(4,5)$. However, there are relatively few reports of PED-IL at L4-L5 $(6,7)$. Therefore, this study aimed to evaluate initial clinical outcomes of PED-IL at L4-L5, compared with those at L5-S1.

\section{Methods}

Since April 2014, the senior author has performed PED-IL, without experience in PED-TF. However, before beginning PED-IL, the senior author observed more than 10 PED-IL procedures, and the first 10 cases were performed under the supervision of an experienced surgeon. Written informed consent was obtained from all patients and our Institutional Review Board approved this study.

\section{Surgical Techniques}

PED-IL was performed with the patient in the prone position on a radiolucent table using C-arm fluoroscopy. An 8-mm skin incision was made at the inferior edge of the superior lamina that was 2 or 10 o'clock to the interlaminar window of the symptomatic side at the affected level in the AP view and parallel to the disc space in the lateral view (Fig. 1). A dilator $(6.9 \mathrm{~mm}$ in diameter) and working sleeve were bluntly inserted for subsequent endoscope insertion. PED was performed using a $25^{\circ}$ endoscope with a $4.1-\mathrm{mm}$ working channel and associated special equipment (Richard Wolf $\mathrm{GmbH}$, Knittlingen, Germany). The ligamentum flavum was resected piece-by-piece using a micro punch under direct visualization using an endoscope. A 3-mm micro rongeur (Tanaka Ika, Tokyo, Japan) was used for additional resection of the ligament. A beveled-type sleeve was placed lateral to the thecal sac or nerve root and slightly rotated for retraction of the thecal sac. The herniated disc was then resected. A Trigger-Flex ${ }^{\circledR}$ bipolar coagulator (Elliquence, Baldwin, NY, USA) was used for hemostasis of the epidural venous plexus. A specially

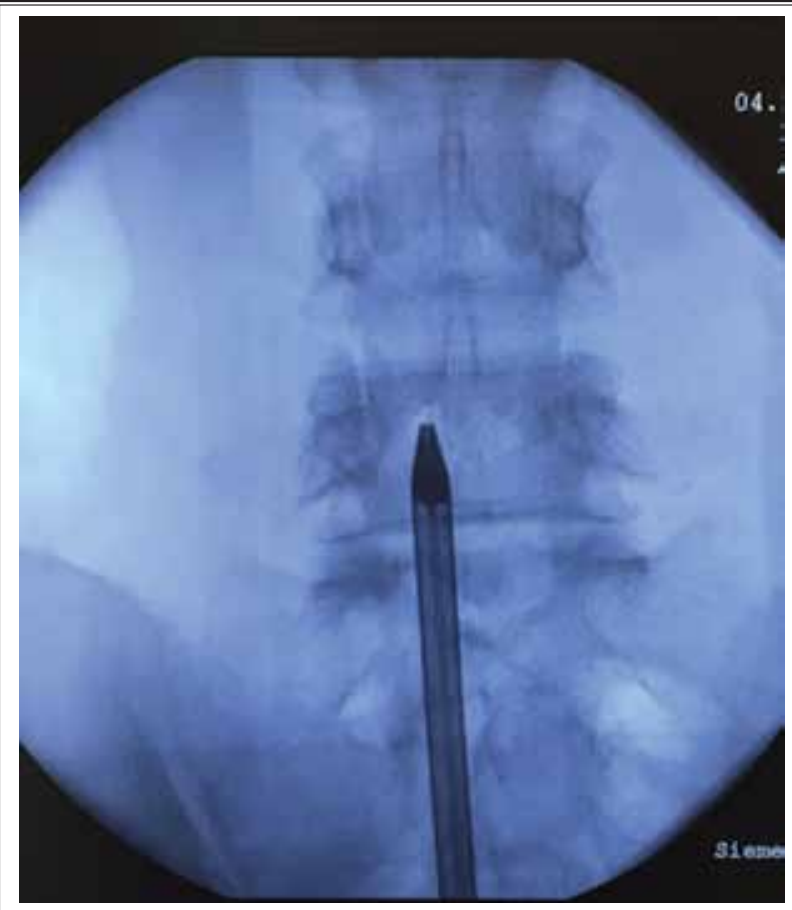

$A$

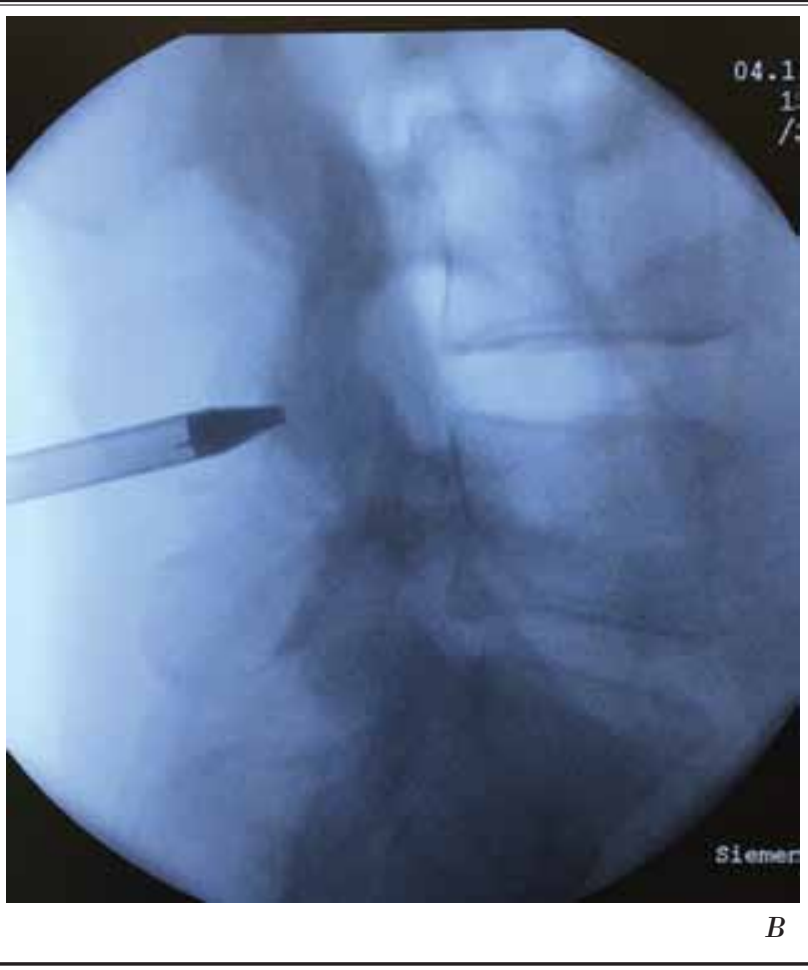

$B$

Fig. 1. Intraoperative fluoroscopic image. The placement of the dilator in the interlaminar window at L4-L5 in the PA view (a) and lateral view (b). The insertion point of the dilator was just above the inferior edge of L4 lamina. 
designed diamond burr system for PED, Primado (Nakanishi, Inc., Kanuma, Japan), was used for laminotomy and medial facetectomy. Particularly at L4-L5, partial laminotomy was necessary in all cases. These cases needed sufficient removal of laminae and ligamentum flavum to confirm the lateral edge of the nerve root. Initially, the herniated disc should be removed via the shoulder of the nerve root, while being careful not to retract the nerve root. After decompressing the nerve root, the nerve root should be slightly retracted, and the residual central part of the herniated disc should be resected (Fig. 2). Removal of a herniated disc via the axilla of the nerve root was sometimes required. Patients with cauda equina syndrome or central large herniation at L4-L5 were excluded as an indication for PED-IL.
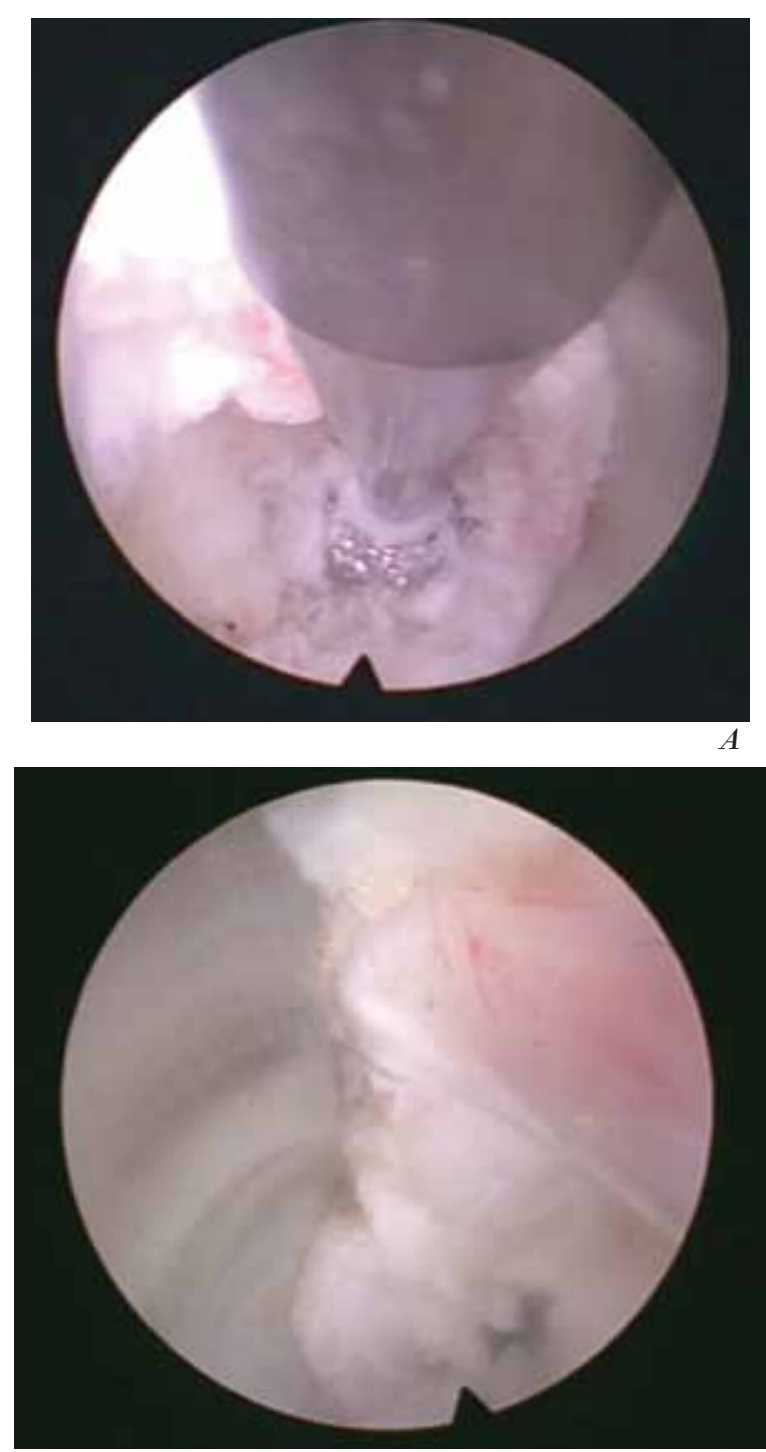

C

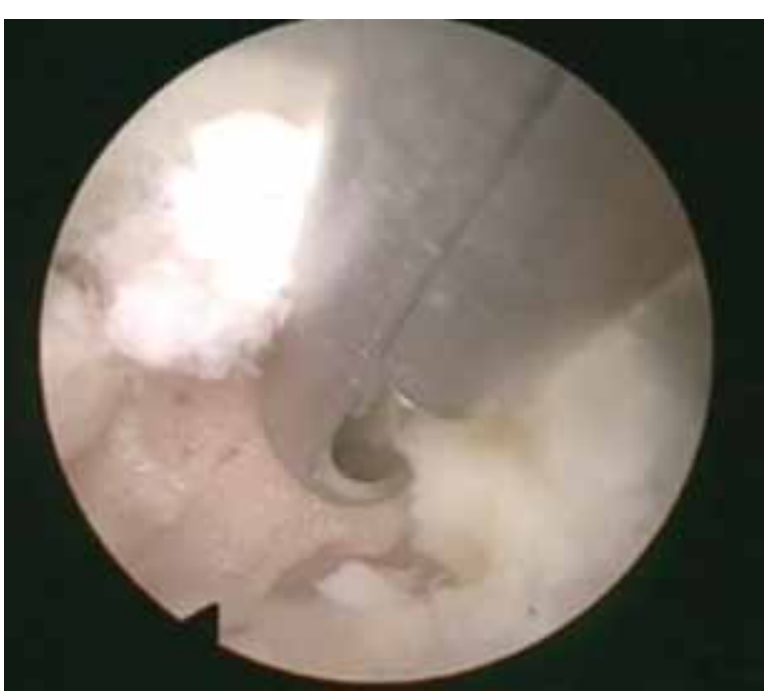

$B$

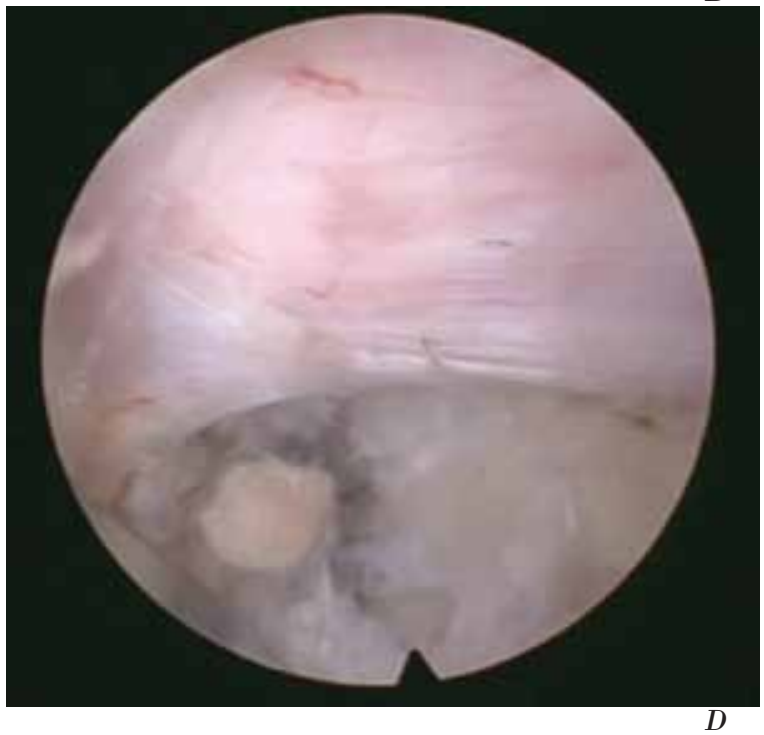

Fig. 2. Intraoperative endoscopic view at the L4-L5 level. Partial resection of laminae using microdiamond burr (a), removing the ligamentum flavum using 3-mm width Micro Rongeur (b), retracting the thecal sac by rotating the sleeve (c), and decompression was confirmed via endoscope (d). 
Variables for analysis included operative time, length of hospital stay, complications, recurrence, blood loss, and incidence of reoperation. Clinical outcomes were assessed according to the modified MacNab criteria. Pre- and postoperative leg pain was measured using a visual analog scale (VAS; 0-10). Operative times at L4-L5 and L5-S1 were compared. Each herniated nucleus pulposus (HNP) was classified as non-migrated or migrated.

Descriptive analysis of group characteristics was performed using JMP version 11.2 software for Macintosh (SAS Institute Inc., Cary, NC, USA). The independent 2-sample t-test and Wilcoxon test were used to compare the clinical outcomes of interlaminar endoscopic lumbar discectomy employed between L4-L5 and L5-S1. A probability value $(P)$ of $<0.05$ was considered to be statistically significant.

\section{Results}

Fifty patients had PED-IL performed. PED-IL was successful in all patients and no case required conversion to open surgery. There were 36 men and 14 women, and the mean age of patients was 40.3 years old (range: 21-59 years). The affected level was 16 cases at L4-L5 and 34 cases at L5-S1. The type of HNP was classified as paracentral contained type (11 at L4-L5 and 20 at L5-S1), central type (2 at L4-L5 and 33 at L5-S1), and migration (3 at L4-L5 and 11 at L5-S1). The mean follow-up period was 15 months (range: 7-22 months). The mean operative time was $71.3 \pm 19.3$ minutes for all cases (range: $41-112$ mins.), $76.1 \pm 16.8$ minutes at L4-L5 (range: 52-102 mins.), and 70.5 \pm 20.1 minutes at L5-S1 (range:41-112 mins.). The operative time was gradually decreased, and the learning curve eventually flattened (Fig. 3). There was no significant difference in operative time between L4-L5 and L5-S1. The operative time was significantly shorter in the non-migration group compared with the migration group (70.95 \pm 24.26 mins. vs. $82.53 \pm 19.27$ mins., respectively; $P<0.05$, t-test) The mean hospital stay was $3.2 \pm 1.6$ days for all cases (range: $2-8$ days), 3.9 1.8 days for the L4-L5 group, and $2.9 \pm 1.4$ days for the L5-S1 group $(P=0.241)$.

The mean VAS score for leg pain decreased from $7.4 \pm 2.0$ (range: $6-10$ ) to $1.3 \pm 1.6$ (range: $0-5$ ). Accord-

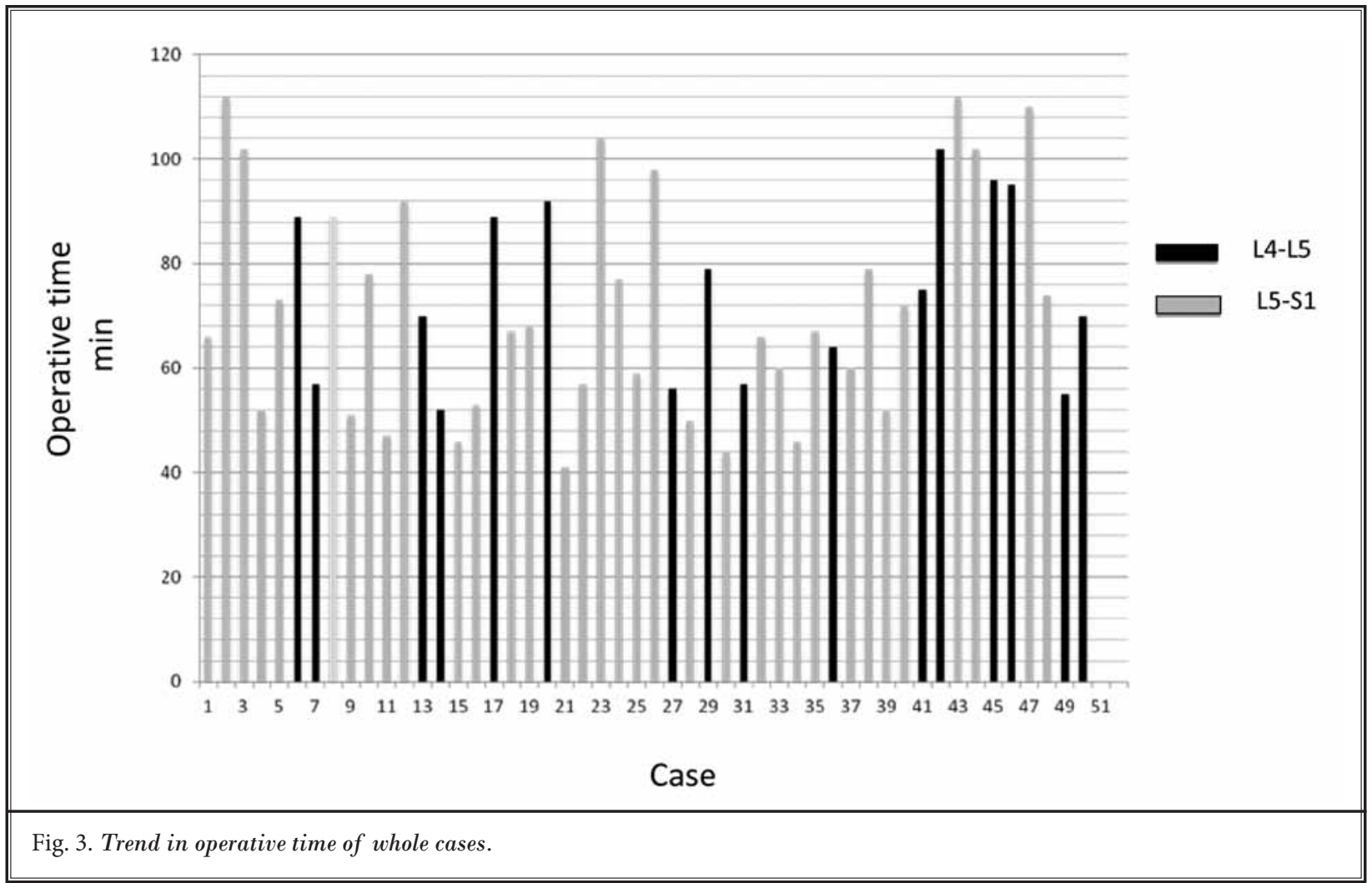


ing to the modified MacNab criteria, 20 cases ( 6 at L4-L5 and 14 at L5-S1) were excellent, 27 (10 at L4-L5 and 17 at L5-S1) were good, one at L5-S1 was fair, and 2 at L5-S1 were poor. Patients rated as fair and poor were further examined postoperatively by magnetic resonance imaging, which showed complete HNP removal. One patient who was rated fair developed a discal cyst postoperatively, which was treated with epidural block and medication. Two nerve root injuries (perineurium tears) occurred at $\mathrm{L} 5 \mathrm{~S} \mathrm{~S} 1$, but did not require repair. These 2 patients complained of moderate numbness at the final follow-up. Wound dehiscence occurred in one case, which was resutured at our outpatient clinic. There was no instance of recurrence or infection, and blood loss was negligible.

\section{Discussion}

There have been numerous reports of PED-TF since its development (2-4). Although the learning curve of PED-TF is reportedly steep, its operative time is shorter than that of PED-IL $(8,9)$.

The operative time in this study was the same as in other reports of initial PED-IL procedures $(7,8)$. There was no significant difference in operative time between the L4-L5 and L5-S1 groups. Factors contributing to an extended operative time were due to the type of HNP: migrated or a giant central herniated disc. There were no complications of nerve root injury at L5-S1. This study showed that initial clinical outcomes of PED-IL at L4-L5 were sufficient and safe, compared with L5-S1.

Yeung and Tsou (10) suggested that all cases could be accessible using the PED-TF approach, even at L5-S1. However, Ruetten et al (4) reported that 95\% of L5-S1 cases were performed using the PED-IL approach. The selection of the PED-TF approach at L5-S1 is controversial because it is sometimes difficult to perform due to a high iliac wing or a small intervertebral foramen and highly migrated intracanalicular HNP. Choi et al (11) reported the efficacy of the foraminoplastic transforaminal technique for a migrated HNP, which included partial resection of the superior articular processes. Although the foraminoplastic technique is difficult to perform, the associated surgical invasiveness is equal to that of medial facetectomy or laminotomy using PED-IL.

PED-IL was performed using a posterior approach with operative field similar to a microscopic view. Several surgeons are familiar with this operative field. In case of a highly migrated HNP, adding laminotomy or medial facetectomy using a micro diamond burr which was specially designed for PED, a sequestrated disc can be removed using PED-IL. Furthermore, a lateral recess stenosis can be decompressed without difficulty, using a diamond burr and micro rongeur (12). Choi et al (3) reported that PED-IL at L5-S1 is preferred for an axillary type or migrated HNP, whereas PED-TF is preferred for a shoulder type or centrally located HNP. This suggestion can be applied at L4-L5. However, central type disc herniation was not a good indicator of PED-IL at L4-L5.

One of the greatest concerns with PED-TF procedures is the possibility of remnant fragments or inadequate decompression of neural tissue. Decompression of a nerve and thecal sac can be confirmed using the interlaminar approach under direct visualization with an endoscope. The greatest advantage of PED-IL is that the operative field is similar to the microscopic operative view.

The limitations of this study were the small number of cases and the short follow-up period. Further studies are required to compare the clinical outcomes of PEDTF and PED-IL at L4-L5. However, this is the first report of initial clinical outcomes of PED-IL at L4-L5 without PED-TF experience. The findings of this study suggest that PED-IL is suitable as a standard method for intracanalicular lumbar disc herniation at L4-L5.

\section{Conclusion}

Initial clinical outcomes of PED-TF at L4-5 were equal to those at L5-S1. PED-IL is suitable as a standard method for endoscopic discectomy at L4-L5.

\section{References}

1. 1. Ruetten S, Komp M, Godolias G. FullEndoscopic interlaminal operation of lumbar disc herniations using new endoscopes and instruments. Orthop Praxis 2005; 10:527-532.

2. Choi KC, Lee JH, Kim JS, Sabal LA, Lee S, Kim H, Lee SH. Unsuccessful percuta- neous endoscopic lumbar discectomy: A single-Center experience of 10228 cases. Neurosurgery 2015; 76:372-780.

3. Choi KC, Kim JS, Ryu KS, Kang BU, Ahn Y, Lee SH. Percutaneous endoscopic lumbar discectomy for $\mathrm{L}_{5}$-S 1 disc herniation: Transforaminal versus inter- laminar approach. Pain Physician 2011; 16:547-556.

4. Ruetten S, Komp M, Merk H, Godolias G. Full-Endoscopic interlaminar and transforaminal lumbar discectomy versus conventional microsurgical technique: A prospective, randomized, con- 
trolled study. Spine 2008; 33:931-939.

5. Choi G, Lee S, Raiturker PP, Lee S, Chae YS. Percutaneous endoscopic interlaminar discectomy for intracanalicular disc herniations at $\mathrm{L}_{5}$-S1 using a rigid working channel endoscope. Neurosurgery 2006; 58:59-68.

6. Ruetten S, Komp M, Godolias G. A new full-Endoscopic technique for the interlaminal operation of lumbar disc herniations using $6 \mathrm{~mm}$ endoscopes: Prospective 2-Year results of 331 patients. Minim Invas Neurosurg 2006; 49:80-87.

7. Bing Wang, Guohua Lü, Alpesh A. Patel, Peigen Ren, Ivan Cheng. An evaluation of the learning curve for a complex surgical technique: The full endoscopic interlaminar approach for lumbar disc herniations. Spine J 2011; 11:122-130.

8. Hsu HT, Chang SJ, Yang SS, Chai CL. Learning curve of full-Endoscopic lumbar disectomy. Eur Spine J 2013; 22:727-733.

9. Lee DY, Lee SH. Learning curve for percutaneous endoscopic lumbar discectomy. Neurol Med Chir (Tokyo) 2008; 9:383-388.

10. Yeung AT, Tsou PM. Posterolateral endoscopic exciseon for lumbar disc herniateon: Surgical technique, outcome, and complications in 307 consecutive cases. Spine 2002; 27:722-731.

11. Choi G, Lee SH, Lokhande P, Kong BJ, Shim CS, Jung B, Kim JS. Percutaneous endoscopic approach for highly migrated intracanal disc herniations by foraminoplastic technique using rigid working channel endoscope. Spine 2008; 33:E508-E515.

12. Komp M, Hahn P, Merk H, Godolias G, Ruetten S. Bilateral operation of lumbar degenerative central spinal stenosis in full-Endoscopic interlaminar technique with unilateral approach: Prospective 2-Year results of 74 patients. J Spinal Disord Tech 2011; 24:281-287. 